\title{
Estimating Bridge Deterioration Age Using Artificial Neural Networks
}

\author{
Aseel Hussein and Abid Abu Tair
}

\begin{abstract}
Deterioration of reinforced concrete bridges is major issue in structural engineering due to the difficulty of estimating or predicting the service life of the bridge. Two types of models were developed to estimate the service life, one deterministic and the other probabilistic. The reliability of these models is questioned since they do not account for the many factors involved. Therefore, for this research artificial neural network (ANN) is used to estimate the deterioration age for RC bridges based on actual deterioration data. Historical records of bridges located in London is used to train and test ANN. Feedforward neural network is designed to be able to estimate the deterioration age. ANN inputs are bridge type, member type, exposure, and defects while the target is the defects age., Design of experiment is conducted to select and monitor the most important parameters that would affect ANN performance. The results were mediocre reflecting the type of data provided in neural network training.
\end{abstract}

Index Terms-Bridge deterioration, artificial neural networks, design of experiment.

\section{INTRODUCTION}

To tackle the issue of degradation, a deterioration model is needed to estimate the service life or degradation age. Several methods were developed to predict the stochastic degradation process of RC bridges. Three main techniques are used for developing the deterioration model. Deterministic and Probabilistic approaches are the most two common type of methods to develop a service life model. These techniques are used frequently, However, they fall short in providing reliable results. Recently, scholars became interested in exploring Artificial Neural Network capability in estimating the deterioration process of bridges. In the age of computers and technology, Artificial Intelligence approach is the trend for many engineering fields, where complexity and variability exist. This research will focus on using Artificial Neural Network to estimate the deterioration age for bridges using historical records.

\section{ARTIFICIAL NEURAL NETWORK (ANN)}

ANN logic is derived from the human brain neural cells function and how these cells are connected to solve complex issues. Neural networks can recognize patterns, classify data, and approximate the solution for a complex non-linear function (Haykin 1999) [1]. Choosing ANN type depends on the type of system and data available to process. Different types of Neural Network exist, depending on the action and desired target the network could be assembled in

Manuscript received May 7, 2018, revised June 13, 2018.

Aseel Hussein and Abid Abu Tair are with the The British University in Dubai (BUID) Dubai, UAE (e-mail: Aseelal89@live.com, abid.abutair@buid.ac.ae) any manners. There are four categorizes of ANN tasks; Fitting and approximation Neural networks, Pattern Recognition and Classification, Clustering Neural Network and Time Series \& Dynamic Neural Network. Two main types of artificial neural networks exist, feed forward neural networks and Recurrent neural networks.

According to Hurtado [2], Neural Networks has shown the ability to examine uncertainty of one dimension in stochastic finite element problems. In a study of concrete bridge decks and life cycle costs, a model of Artificial neural networks with genetic algorithm was used. The type of ANN is a feed forward network with backpropagation learning function. The designed neural network has three layers with sigmoid transfer function for hidden layer and linear function for the output layer.

Another study conducted by $\mathrm{Bu}$ et al (2012) [3], where integrated method for deterioration model used Elman Neural Networks (ENN) to substitute the regression function in predicting the deterioration patterns. ENN are a type of feedforward network with layers of recurrent connections. The integrated method used the probabilistic time and state-based models. ENN is incorporated in statebased model to generate the bridge members condition rating. The study showed good results for ENN, nevertheless, the authors recommended more case studies to confirm the reliability of neural networks. In another study, Lee at el. (2010) [4], Elman neural network has been used instead of time delayed neural network. According to this work, TDNN also a feedforward network with time sequence inputs, has produced illogical pattern and irregular noise pattern, which caused poor training for the data. Thus, ENN as recurrent neural network has advantage over feedforward neural network. This research has provided a comparison between feedforward neural network results and recurrent network.

In a study by Chun et al. (Chun, Yamashita \& Furukawa 2015) [5], multilayer perceptron feedforward NN is used to quantify the damage severity in bridges. The neural network is first trained for FEM (Finite Element Method) analysis and then the resulted were confirmed. The study acknowledges the advantages of using artificial neural networks with large accumulative data. Kabir et al, used Multilayer Perceptron (MLP) as pattern recognizer and classifier for texture analysis to evaluate the bridge structural damage (Kabir, Rivard \& Ballivy 2008) [6].

Huang (2010) [7] has developed ANN model for bridge deterioration where eleven factors are used as input and five condition state for output. Multilayer Perceptron with backpropagation is used to predict the condition state. Hung has used trial and error to determine the best number of hidden layers and hidden neurons. "The ANN prediction model reaches classification rates of $84.66 \%$ and $75.39 \%$ for 
the training sets and the testing sets, respectively" (Huang, 2010). Furthermore, ANOVA is used to check the significance of maintenance history for condition states 1-2 and 2-3. In his study, Hung claims that Markov Chain approach used by BMS, Bridge Management system is unrealistic and inaccurate for prediction of bridges deck deterioration and propose Artificial Neural Network with Multilayer Perceptron (MLP) as good tool for determining the deterioration rate and the best time for maintenance cycles. Furthermore, ANN used as pattern classification tool which categorize the inputs and output into similar classifications with utilizing Back-propagation tool to substitute for missing data. MATLAB programs were used and developed to construct the ANN prediction model.

\section{DATABASE}

Historical records of Inspections performed of more than 400 reinforced concrete bridges built in London, United Kingdom, between $1880 \& 1960$ is used in this study [8][12]. The first record of inspection was registered in the late of 1920s and continued till the 1980s. Sixty years of defect histories is included in the database of four hundred bridges. Some of these bridges were inspected several times throughout the years and other bridges were only inspected once. Therefore, there is no pattern of inspection and recording. The current dataset has huge and massive information about the 400 bridges. So, Data Analysis is necessary step when handling a large quantity of information. The raw gathered data are scattered and random with many inadequate information. Therefore, the database has undergone extensive analysis to obtain the best information needed for estimating the defects' age of concrete bridges. This step is vital for using Neural Network and obtaining valid results. Since Feedforward Neural Network is selected for this research, the quality of the dataset would directly impact the performance due to function fitting analysis.

After the analysis, 220 rows of Historical data are ready to be used for neural networks modelling. The data is still scattered and have a degree of randomness because some of the same factor scenarios do not have a similar corresponding defect age. Forty-three degradation scenarios are used for the deterioration modelling.

\section{DATA INPUT FOR ANN AND DETERIORATION}

Input data is very important because it impacts the generalisation ability of ANN. In this study, the acquired bridges database has several parameters to investigate the model, such as the exposure condition, defect type, defected member type and bridge type. These factors are used as input data to develop a neural network model as shown in Table I, while the age of bridge based on the recorded defect is used as the target. Artificial Neural Networks can be built in unlimited number of ways. ANN architecture could be designed in many shapes and types relying on many factors, such as; the number of input neurons, number of output neurons, number of hidden layers, number of hidden neurons, learning algorithm, transfer function, types of connections between all these neurons and etc. Nevertheless, there are four important parameters to design a network as demonstrated in Table II.

TABLE II: ANN DATA

\begin{tabular}{|l|l|l|l|l|}
\hline \multicolumn{4}{|c|}{ ANN Input } & ANN Target \\
\hline $\begin{array}{l}\text { Bridge } \\
\text { Type }\end{array}$ & $\begin{array}{l}\text { Member } \\
\text { Type }\end{array}$ & $\begin{array}{l}\text { Exposure } \\
\text { Condition }\end{array}$ & $\begin{array}{l}\text { Defect } \\
\text { Type }\end{array}$ & Age of the Defect \\
\hline
\end{tabular}

\begin{tabular}{|l|l|l|}
\multicolumn{4}{|c|}{ TABLE II: ANN FACTORS } \\
\hline Factors & Low-Level & High-Level \\
\hline Learning Algorithm & LM & GDX \\
\hline Number of hidden layers & 1 & 2 \\
\hline Number of Neurons & 10 & 20 \\
\hline Transfer Function & Tansig & Purelin \\
\hline
\end{tabular}

To test these parameters, a design of experiment is conducted to ensure adequate and control setting for the experiment. The definition of experiment (DOE) is systematic tests conducted in controlled conditions to determine the factors effecting the output of a system and to examine if a theory or hypothesis is correct. One of the most common techniques for DOE and used to evaluate the interaction between factors. There are two levels for each factor, high and low levels. Factors are referred to as K So, the sample size $\mathrm{N}$ is equal to $2 \mathrm{k}$. The samples of the full factorial scheme are a part of the sample space where change occur one at a time. Therefore, effect of each factor over the response variable will not be confounded with other factors. Factorial design of 24 is used for this research to test Artificial Neural network performance. Four factors with two levels are chosen as parameters to study their influence on modelling degradation. The experiment is conducted three times to eliminate noisiness and to ensure minimum error or misreading. A total of 48 experiment conducted using MATLAB with 16 combinations replicated three times.

\section{RESULTS}

The results of feed forward neural network for estimating defects age is investigated. DOE test the parameters for Artificial neural network and examine their impact on achieving the desired target. Factorial design of (24) is used on ANN design parameters with the defects age as the target. A total number of $16 \mathrm{ANN}$ is designed using MATLAB, each of these scenarios was replicated for three times to limit errors or noises. The total runs were 48 and the results are evaluated using Mean Square Error (MSE), regression and error histogram. These tools measure the difference between targets (true age) and outputs (ANN age). Fig. 1 shows MSE results for 16 experiments. Some models had lower MSE which indicates better performance of ANN. The results range between 0.02-0.05 for mean square error. This also shows that some of the four factors had significant impact of neural network outcome. Regression of the 16 models is demonstrated in Fig. 2. The higher the value of regression, the better result of neural network. The type and quality of data inserted to ANN might have an impact on the regression result of this experiment.

After running analysis of variance on the mean square 
error for 48 experiments, hidden neuron is a significant factor with $\mathrm{p}$-value $0.002<0.05$. According to the main effect plot, Fig. 6.17, ten neurons is better for ANN outcome than twenty neurons. The significance of the factor means there is a difference between ten neurons MSE and twenty neurons MSE. The mean square error for $10 \mathrm{H}$ Neurons is 0.034 while twenty neurons have higher MSE of 0.0405 . The other factors did not achieve P-value $<0.05$. This means that two levels; low and high for learning algorithm, hidden layer and transfer function have the similar impact on ANN performance, Hence the difference between the effects of the two options is insignificant.

ANOVA outcomes provide another insight on the influence of the design parameters on Artificial Neural network. When the factor is not significant, it means the two options has similar impact on mean square error results. In this research, there are three factors that came as insignificant, Learning algorithm, Hidden layer and Transfer function.

Levenberg Marquardt (LM) and Gradient descent (GD) are two backpropagation learning technique and their similar impact on MSE values indicates similar training method. Nevertheless, LM is faster learning technique than GD. This research finding is very important and unique since there is no previous literature review on the matter. This insight could be a valid through all the cases or only true for this experiment. Hidden layer is also found as insignificant factor which means that one and two hidden layers had the same impact on MSE. Two hidden layers are usually associated with overfitting if the network is small, but in this case the results are similar with one hidden layer. This finding is true for this experiment, but the previous knowledge indicates that the two hidden layers have different impact on the results. This indicates other factors such as Feed forward network and type of data might have caused this finding. Output Transfer function is to refine the output values, and in this experiment using Linear and Hyperbolic Tangent Sigmoid has similar impact on mean square error for this experiment. Linear function has better impact on the results and its supposedly is a part of universal approximation function of neural network. However, it had similar impact with sigmoid function on the output.

\section{Mean Square Error}

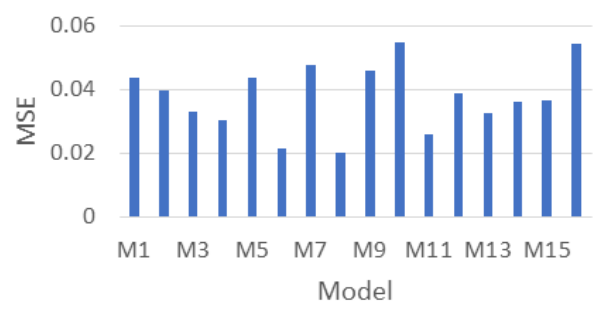

Fig. 1. Histogram of MSE for 16 models.

Hidden Neurons is the only significant factor where ten neurons have better impact on the results than twenty neurons. Determining the suitable number of hidden neurons is a matter of trial and error. As mentioned in methodology section, each case has its own adequate number of neurons, therefore there are no standard or rules that the findings can be compared to at this stage.

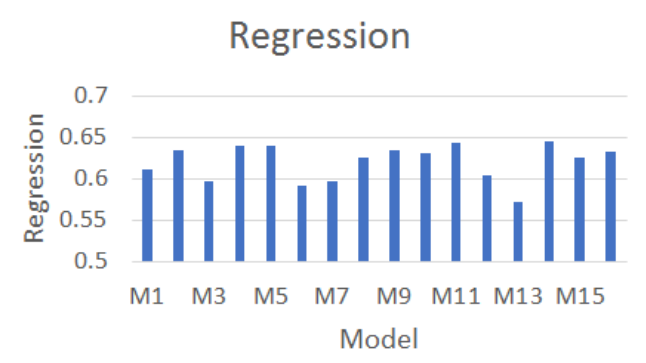

Fig. 2. Histogram of regression for 16 models.

\section{CONCLUSION}

The neural network results reveal the difference between the original data for deterioration age and the neural network generated deterioration age. Although the difference varied between the sixteen models, most of the models had similar range in the similarity and differences. Three validation methods were taken to measure the performance of ANN, Mean Square Error, Regression, and Error histogram. MSE values ranging between 0.02-0.05 and Levenberg Marquardt training function has faster learning rate than Gradient descent The performance figure shows that training, validation, and testing sets converge at the early Epoch and then start to diverge at the end, insinuating overfitting for the network, but the learning function stop the network from overfitting and record the results. Regression analysis is at best $65 \%$ which shows good results but with random values between the targets and outputs. The components of neural networks are not responsible of the random values, increasing the number of hidden layers or neurons could result in generalization of the output. The inconsistency and the randomness of the original data caused the mediocre results.

\section{REFERENCES}

[1] S. Haykin, "Neural networks a comprehensive foundation," 2nd ed. Delhi: Pearson Education, 1999.

[2] J. E. Hurtado, D. A. Alvarez, and A. H. Barbat, "Monte carlo analysis of structural systems using neural networks," Monte Carlo Simulation, pp. 265-271, 2001.

[3] G. Bu, J. Lee, H. Guan, Y. Loo, and M. Blumenstein, "Implementation of Elman neural networks for enhancing reliability of integrated bridge deterioration model," Australian Journal of Structural Engineering, vol. 15, no. 1, 2012.

[4] J. lee, H. Guan, Y. C. Loo, M. Blumenstein, and W. X. Ping. (2010). Modelling long-term bridge deterioration at structural member level using artificial intelligence techniques. [Online]. Available. http://10.4028/www.scientific.net/amm.99-100.444

[5] P. Chun, H. Yamashita, and S. Furukawa, "Bridge damage severity quantification using multipoint acceleration measurement and artificial neural networks," Shock and Vibration, vol. 2015, pp. 1-11, 2015.

[6] S. Kabir, P. Rivard, and G. Ballivy, "Neural-network-based damage classification of bridge infrastructure using texture analysis," Canadian Journal of Civil Engineering, vol. 35, no. 3, pp. 258-267, 2008.

[7] Y. H. Huang, "Artificial neural network model of bridge deterioration," Journal of Performance of Constructed Facilities, vol. 24, no. 6, pp. 597-602, 2010

[8] S. R. Rigden, A. I. Abu-Tair, and E. Burley, "Predicting future performances of concrete bridges using long term bridge inspection records," in Proc. 1993 International Conference on Structural Faults and Repairs, Edinburgh, 1993, vol. 1, pp. 43-46.

[9] A. H. Christer, D. F. Redmond, S. R. Rigden, and A. I. Abu-Teir, "Modeling deterioration and maintenance of concrete structures," in 
Proc. International Conference of Operation Research, Lisbon, July 1993.

[10] S. R. Rigden, S. M. A. Tajalli, and A. I. Abu-Teir, "Long term performance of concrete bridges," Construction Repair, vol. 10, no. 4 1996, pp. 36-38.

[11] S. R. Rigden, S. M. A. Tajalli, and A. I. Abu-Teir, "Service life prediction of concrete bridges," in Proc. International Congress Concrete in the Service of Mankind, 1996, pp. 705-714.

[12] C. McParland, A. Abu-Tair, A. Nadjai and J. F. Lyness, "Modeling deterioration of concrete bridges using a large database of inspection records," Structural Faults and Repair - 2001 - Extending the Life of Bridges, 2001.

Aseel Hussein is a graduate engineering student at the British University in Dubai. She has a civil engineering degree from American University of Sharjah and 5 years experinece working in construction field.

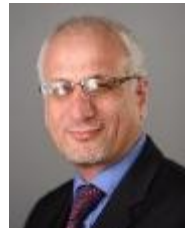

Abid UI Razzaq Abu-Tair is a professor of structural engineering at the British University in Dubai and visiting research scholar of the Manchester School of Mechanical, aerospace and civil engineering. $\mathrm{He}$ is a chartered civil engineer of the UK Engineering Council and a fellow of the Institution of civil engineers in the UK. He is also a senior fellow of the UK Higher Education Academy, a qualification of teaching and learning and educational management. He was awarded the Henri Adam Diploma by the Institution of Structural Engineers UK and the silver award by the Permanent Way Institute UK for paper published in their journals.

He worked at three UK Universities and three in the Middle East. His major research areas relate to the structural performance, sustainability and asset management with emphasis on durability and rehabilitation of concrete structures 